\title{
Human Herpesvirus 8-Negative and Epstein-Barr Virus-Positive Effusion- Based Lymphoma in a Patient with Human Immunodeficiency Virus
}

\author{
Jung-Woo Choi · Younghye Kim \\ Ju-Han Lee · Young-Sik Kim \\ Department of Pathology, Korea University Ansan \\ Hospital, Korea University College of Medicine, \\ Ansan, Korea \\ Received: May 12, 2015 \\ Revised: June 2, 2015 \\ Accepted: June 3, 2015 \\ Corresponding Author \\ Young-Sik Kim, MD, PhD \\ Department of Pathology, Korea University Ansan \\ Hospital, Korea University College of Medicine, 123 \\ Jeokgeum-ro, Danwon-gu, Ansan 15355, Korea \\ Tel: +82-31-412-5321 \\ Fax: +82-31-412-5324 \\ E-mail: apysk@korea.ac.kr
}

\begin{abstract}
A 39-year-old man infected with human immunodeficiency virus (HIV) was admitted to our hospital because of sudden onset of chest pain. Chest radiography revealed pneumothorax of the right lung. Computed tomographic scans disclosed a 5.8-cm-sized emphysematous bulla in the right middle lobe of the lung. Histologically, the wedge-resected lung showed medium to large atypical cells within the bullous cavity of the Pneumocystis jirovecii pneumonia, without solid mass formation. These atypical cells were confirmed to be large B-cell lymphoma, Epstein-Barr virus-positive and human herpesvirus 8-negative. Therefore, this case was not diagnosed as primary effusion lymphoma, but effusion-based lymphoma arising in an emphysematous cavity of an HIVinfected patient. This type of effusion-based lymphoma has never been reported, and, although rare, it should be noted in order to clinically diagnose this lymphoma.
\end{abstract}

Key Words: Effusion-based lymphoma; Human herpesvirus 8; Epstein-Barr virus; HIV
Primary effusion lymphoma (PEL) is the only subtype of effusion-based lymphoma (EBL) listed on the 2008 World Health Organization (WHO) classification, and it is specified as a large B-cell lymphoma that presents as serous effusions without a detectable tumor mass. ${ }^{1}$ PEL represents one of the specific lymphomas that occur in human immunodeficiency virus (HIV)-positive patients, accounting for less than $3 \%$ of all HIV-related lymphomas. ${ }^{2}$ PEL cells are usually negative for pan-B-cell markers and often reveal a consistent dual herpesvirus infection with both human herpesvirus 8 (HHV8) and Epstein-Barr virus (EBV). Recently, HHV8-negative EBL has emerged as a distinct entity, often associated with older patient age, clinical history of fluid overload, and a universal expression of pan-B-cell markers. ${ }^{3,4} \mathrm{~Pa}$ tients with HHV8-negative EBL tend to be positive for the hepatitis $C$ virus, but negative for HIV and EBV infection. Meanwhile, HHV8-positive solid lymphomas without lymphomatous effusions are considered extracavitary variants of PEL, which have been validated via gene expression profiling and proteomic analyses. ${ }^{5}$ In this context, we report herein another unique case of HHV8-negative and EBV-positive EBL, which developed from the bullous cavity of Pneumocystis jirovecii pneumonia (PJP) in a clinical background of HIV infection.

\section{CASE REPORT}

A 39-year-old man visited our hospital because of two enlarging dusky-colored patches on his chin and forehead, both of which appeared 1 month prior to his visit. Both skin lesions were diagnosed as Kaposi sarcoma, and the patient was diagnosed to be HIV positive. Two months later, he experienced sudden onset of chest pain and difficulty breathing. Laboratory tests showed high levels of white blood cells $(10,600 / \mu \mathrm{L})$ with $73.5 \%$ granulocytes and serum lactate dehydrogenase of $679 \mathrm{IU} / \mathrm{L}$. The patient's CD4 T-cell count had decreased to 19 cells $/ \mu \mathrm{L}$. Hepatitis $\mathrm{A}, \mathrm{B}$, or $\mathrm{C}$ viruses were not detected. Computed tomographic scans of his chest revealed a 5.8-cm emphysematous bulla in the right middle lobe of his lung, while the left lobe showed diffuse ground glass opacities, suggestive of interstitial pneumonia (Fig. 1A). The patient underwent wedge resection of the lung, which grossly revealed a multilocular bullous cavity surrounded by whitish pneumonic consolidation (Fig. 1B). Microscopically, aggregates of atypical lymphoid cells were floating in the bullous cavity without forming solid masses. These cells showed medium to large pleomorphic nuclei with prominent nucleoli and basophilic cytoplasm, revealing plasmablastic features (Fig. 2). Th- 
rough immunohistochemistry, tumor cells were found to exhibit a non-germinal center B-cell nature showing positivity for CD20, CD79a, and MUM1 but negativity for CD3, CD10, BCL6,
CD30, and CD138. The Ki-67 labeling index reached up to 80\%-90\%. Tumor cells were positive for EBV-encoded RNA in situ hybridization, EBV latent membrane protein-1 (LMP1),
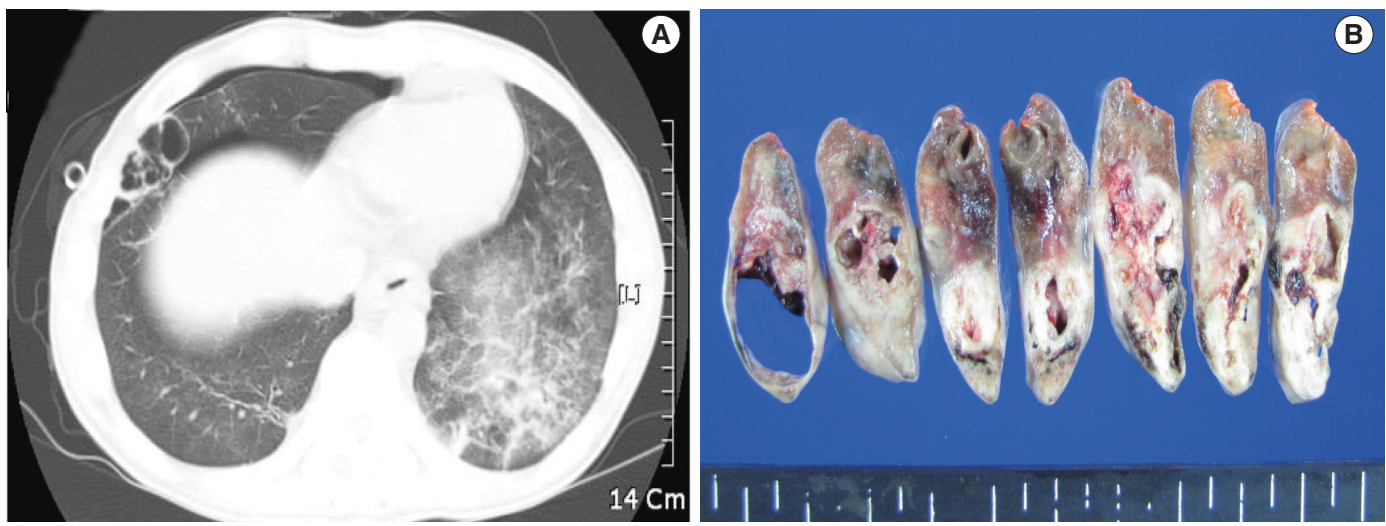

Fig. 1. A computed tomographic scan (A) and serial lung sections (B) displaying a 5.8-cm multilocular emphysematous bulla surrounded by pneumonic consolidation in the right middle lobe of the lung. The left lung shows diffuse ground glass opacities, which is consistent with interstitial pneumonia.
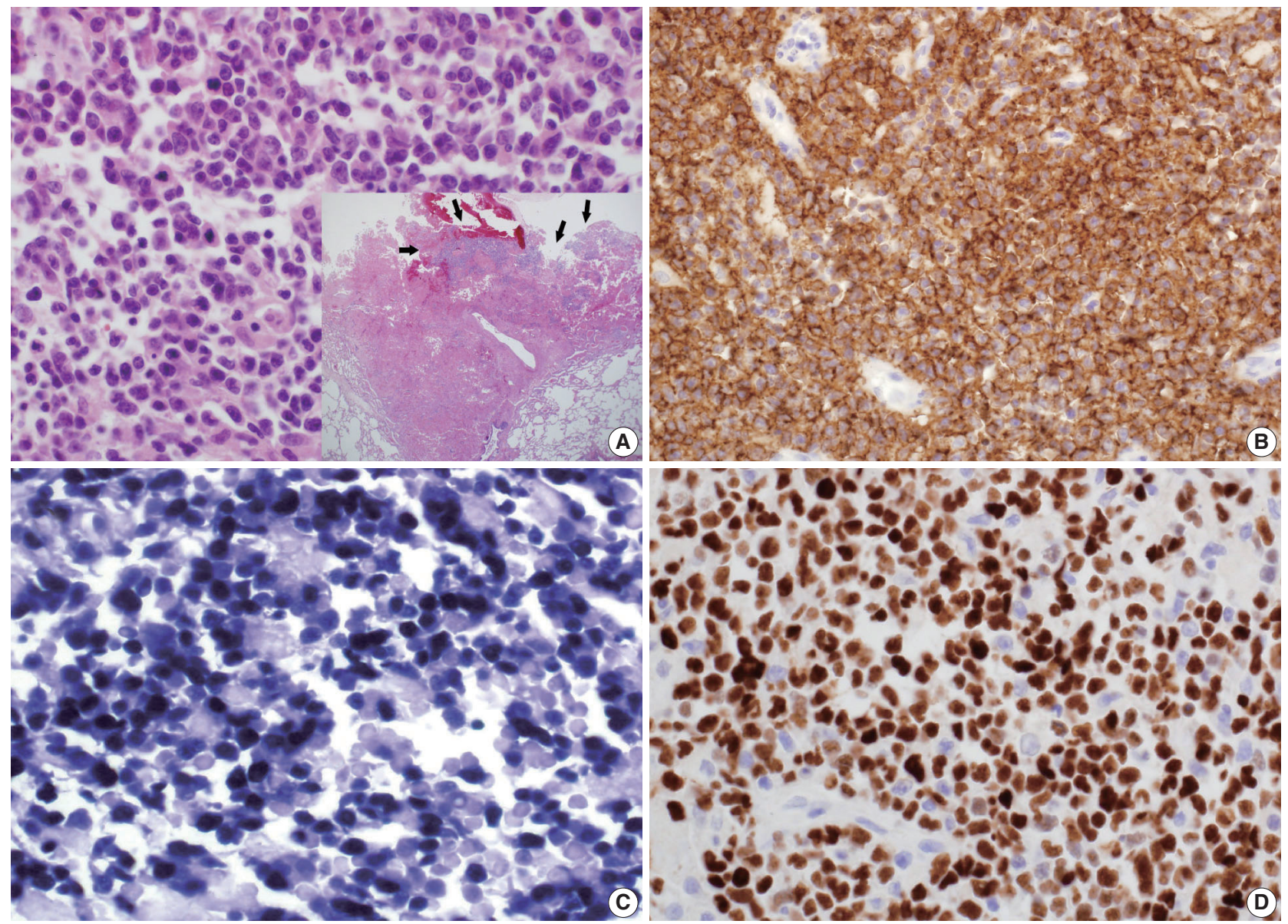

Fig. 2. Lymphoma cells have large eccentric pleomorphic nuclei and basophilic cytoplasm, showing plasmablastic features (A). Proliferating and necrotic tumor cells (arrows) are located in the bullous cavity, representing effusion-based nature (A inset). Tumor cells are positive for CD20 immunohistochemistry (IHC) (B), Epstein-Barr virus (EBV) in situ hybridization (C) and also EBV nuclear antigen-2 IHC (D). 
Table 1. Main differential diagnoses of this case

\begin{tabular}{|c|c|c|c|c|}
\hline & Present case & PEL & HHV8-negative EBL & $\begin{array}{l}\text { DLBCL associated with } \\
\text { chronic inflammation }\end{array}$ \\
\hline Age at diagnosis & $39 \mathrm{yr}$ & Young or middle-aged & Old (median, $70 \mathrm{yr}$ ) & Old (median, 65-70 yr) \\
\hline Underlying condition & Pneumocystis jirovecii pneumonia & $\mathrm{ND}$ & Fluid overload & Pyothorax or chronic pleuritis \\
\hline Site of involvement & Pneumonic bullae & Body cavities & Body cavities & Body cavities \\
\hline Immunophenotype & $\begin{array}{l}\text { CD20(+), CD79a(+), } \\
\text { MUM1(+), CD138(-), CD30(-) }\end{array}$ & $\begin{array}{l}\text { CD20(-), CD79a(-), } \\
\text { CD138(+), CD30(+/-) }\end{array}$ & $\begin{array}{l}\text { CD20(+), CD79a(+), } \\
\text { CD138(+/-), CD30(+/-) }\end{array}$ & $\begin{array}{l}\text { CD20(+/-), CD79a(+/-), } \\
\text { MUM1(+), CD138(+), CD30(+/-) }\end{array}$ \\
\hline Viral infection & $\begin{array}{l}\mathrm{HIV}(+), \mathrm{HHV}(-) \\
\operatorname{EBV}(+, \text { latency III), } \operatorname{HCV}(-)\end{array}$ & $\begin{array}{l}\mathrm{HIV}(+), \mathrm{HHV} 8(+) \\
\mathrm{EBV}(+, \text { latency II), HCV(-) }\end{array}$ & $\begin{array}{l}\operatorname{HIV}(-), \operatorname{HHV} 8(-) \\
\operatorname{EBV}(-), \operatorname{HCV}(+, 26.5 \%)\end{array}$ & $\begin{array}{l}\operatorname{HIV}(-), \operatorname{HHV} 8(-) \\
\operatorname{EBV}(+, \text { latency III), HCV }(-)\end{array}$ \\
\hline Prognosis & Unknown & $\begin{array}{l}\text { Unfavorable } \\
\text { (median survival, }<6 \mathrm{mo} \text { ) }\end{array}$ & $\begin{array}{l}\text { Variable } \\
\text { (median survival, } 10 \text { mo) }\end{array}$ & $\begin{array}{l}\text { Unfavorable } \\
\text { (5-yr survival, 20\%-35\%) }\end{array}$ \\
\hline
\end{tabular}

PEL, primary effusion lymphoma; HHV8, human herpesvirus 8; EBL, effusion-based lymphoma; DLBCL, diffuse large B-cell lymphoma; ND, not described; $\mathrm{HIV}$, human immunodeficiency virus; $\mathrm{EBV}$, Epstein-Barr virus; $\mathrm{HCV}$, hepatitis $\mathrm{C}$ virus.

EBV nuclear antigen-2 (EBNA-2), but negative for HHV8. The background bullous cavity and adjacent alveolar cavities were filled with frothy exudates containing Pneumocystis jirrovecii clearly visible after application of Gomori methenamine silver stain. Therefore, the patient was diagnosed with EBL, not PEL, arising in the PJP-associated cavitary lung lesion. He was treated with highly active antiretroviral therapy along with anti-microbes for 6 months, followed by chemotherapy.

\section{DISCUSSION}

This is a unique case of EBL occurring in the PJP-associated bullous cavity of the lung. The differential diagnosis of this case includes HHV8-negative EBL, PEL, and diffuse large B-cell lymphoma (DLBCL) associated with chronic inflammation (Table 1). While PEL is the only subtype of EBL listed in the 2008 WHO classification, HHV8-negative EBL has been steadily considered a separate entity, because the demographics, immunophenotype, and clinical outcome of HHV8-negative EBL are inconsistent with PEL., ${ }^{3,4}$ In contrast to this case, PEL is invariably positive for HHV8, and usually negative for pan-B-cell markers. ${ }^{1}$ Compared to HHV8-negative EBL, this case did not show any clinical condition of fluid overload such as cirrhosis, protein-losing enteropathy, or cardiac disease. In addition, the tumor in this case developed in the setting of both HIV and EBV positivity, while negative for the hepatitis $C$ virus, which is the reverse of HHV8-negative EBL. With regard to the tumor's origin, the present case is unique in that all previously reported cases of EBL involved pleural, peritoneal, or pericardial cavities, except for one, which was detected from scrotal effusion fluids. ${ }^{6}$ Although our case most closely resembles DLBCL associated with chronic inflammation, in that it is a lymphoid neoplasm often involving the body cavity and occurring in a condition of chronic inflammation and EBV infection, DLBCL forms a detectable tumor mass and typically develops after a long-standing inflammation, usually over 10 years. ${ }^{7}$ It is unclear whether this particular tumor should be considered a previously undescribed subtype of DLBCL, or whether it corresponds with a rare variant of HHV8-negative EBL or DLBCL associated with chronic inflammation.

Interestingly, this tumor was accidentally discovered in the pneumothorax as a complication of PJP. The incidence of pneumothorax in HIV-positive patients is approximately $2 \%$, but can reach up to $9 \%$ in those with PJP. ${ }^{8,9}$ It has been reported that chronic inflammation and localized immunosuppression may enhance the clonal evolution of EBV-infected lymphocytes. ${ }^{10}$ DLBCL cells in HIV-positive patients have been known to express the interleukin-6 (IL-6) receptor, which can respond to the IL-6 produced by adjacent non-neoplastic cells in a paracrine manner. ${ }^{11}$ In addition, similar to DLBCL associated with chronic inflammation, the tumor cells in this case revealed EBV latency type III (LMP1-positive, EBNA-2-positive) infection, which represents the most complete form of latent gene expression. ${ }^{12,13}$ This finding is in contrast to PEL, in which the tumor cells are infected with the more restricted EBV latency type II (LMP1positive, EBNA-2-negative) infection. ${ }^{14}$

In conclusion, the present case is a rare instance of HHV8-negative and EBV-positive EBL in an HIV-positive patient. This type of clinical presentation has never been described previously, and it should be kept in mind in order to diagnose occult lymphomas.

\section{Conflicts of Interest}

No potential conflict of interest relevant to this article was reported. 


\section{Acknowledgments}

This work was supported by Basic Science Research Program through the National Research Foundation of Korea (NRF) funded by the Ministry of Science, ICT and Future Planning (grant number: 2013 R1A1A1058146).

\section{REFERENCES}

1. Said J, Cesarman E. Primary effusion lymphoma. In: Swerdlow SH, Campo E, Harris NL, et al., eds. WHO classification of tumours of haematopoietic and lymphoid tissues. 4th ed. Lyon: IARC Press, 2008; 260-1.

2. Raphael M, Said J, Borisch B, Cesarman E, Harris NL. Lymphomas associated with HIV infection. In: Swerdlow SH, Campo E, Harris $\mathrm{NL}$, et al. eds. WHO classification of tumours of haematopoietic and lymphoid tissues. 4th ed. Lyon: IARC Press, 2008; 340-2.

3. Alexanian S, Said J, Lones M, Pullarkat ST. KSHV/HHV8-negative effusion-based lymphoma, a distinct entity associated with fluid overload states. Am J Surg Pathol 2013; 37: 241-9.

4. Wu W, Youm W, Rezk SA, Zhao X. Human herpesvirus 8-unrelated primary effusion lymphoma-like lymphoma: report of a rare case and review of 54 cases in the literature. Am J Clin Pathol 2013; 140: 258-73.

5. Carbone A, Volpi CC, Caccia D, et al. Extracavitary KSHV-positive solid lymphoma: a large B-cell lymphoma within the spectrum of primary effusion lymphoma. Am J Surg Pathol 2013; 37: 1460-1.

6. Nakamura Y, Tajima F, Omura H, Ishiga K, Kawatani T, Murawaki Y. Primary effusion lymphoma of the left scrotum. Intern Med 2003; 42: 351-3.
7. Iuchi K, Aozasa K, Yamamoto S, et al. Non-Hodgkin's lymphoma of the pleural cavity developing from long-standing pyothorax: summary of clinical and pathological findings in thirty-seven cases. Jpn J Clin Oncol 1989; 19: 249-57.

8. McClellan MD, Miller SB, Parsons PE, Cohn DL. Pneumothorax with Pneumocystis carinii pneumonia in AIDS: incidence and clinical characteristics. Chest 1991; 100: 1224-8.

9. Sepkowitz KA, Telzak EE, Gold JW, et al. Pneumothorax in AIDS. Ann Intern Med 1991; 114: 455-9.

10. Copie-Bergman C, Niedobitek G, Mangham DC, et al. Epstein-Barr virus in B-cell lymphomas associated with chronic suppurative inflammation. J Pathol 1997; 183: 287-92.

11. Emilie D, Coumbaras J, Raphael M, et al. Interleukin-6 production in high-grade B lymphomas: correlation with the presence of malignant immunoblasts in acquired immunodeficiency syndrome and in human immunodeficiency virus-seronegative patients. Blood 1992; 80: 498-504.

12. Cohen JI, Bollard CM, Khanna R, Pittaluga S. Current understanding of the role of Epstein-Barr virus in lymphomagenesis and therapeutic approaches to EBV-associated lymphomas. Leuk Lymphoma 2008; 49 Suppl 1: 27-34.

13. Fukayama M, Ibuka T, Hayashi Y, Ooba T, Koike M, Mizutani S Epstein-Barr virus in pyothorax-associated pleural lymphoma. Am J Pathol 1993; 143: 1044-9.

14. Horenstein MG, Nador RG, Chadburn A, et al. Epstein-Barr virus latent gene expression in primary effusion lymphomas containing Kaposi's sarcoma-associated herpesvirus/human herpesvirus-8. Blood 1997; 90: 1186-91. 